\title{
Probing the Lubrication of Shear-Induced Self- Assembled Layer on Amorphous Carbon Films in Methane Atmosphere
}

\author{
Lin Chen
}

Yanshan University

Jian Wu

Lanzhou Institute of Chemical Physics State Key Laboratory of Solid Lubrication

Zhibin Lu ( $\square$ zblu@licp.cas.cn )

Lanzhou Institute of Chemical Physics https://orcid.org/0000-0003-3145-2934

\section{Lunlin Shang}

Lanzhou Institute of Chemical Physics State Key Laboratory of Solid Lubrication

\section{Guangan Zhang}

Lanzhou Institute of Chemical Physics State Key Laboratory of Solid Lubrication

\section{Qunji Xue}

Lanzhou Institute of Chemical Physics State Key Laboratory of Solid Lubrication

\section{Research Article}

Keywords: Amorphous carbon film, Tribology, Hertzian elastic contact model, first principles calculations.

Posted Date: August 20th, 2021

DOI: https://doi.org/10.21203/rs.3.rs-813504/v1

License: (1) This work is licensed under a Creative Commons Attribution 4.0 International License.

Read Full License

Version of Record: A version of this preprint was published at Tribology Letters on January 10th, 2022.

See the published version at https://doi.org/10.1007/s11249-021-01554-3. 


\title{
Probing the lubrication of shear-induced self-assembled layer on amorphous
}

carbon films in methane atmosphere

\author{
Lin Chen ${ }^{\mathrm{c}}$, Jian Wu ${ }^{\text {a }}$, Zhibin Lu ${ }^{\text {a, b,* }}$, Lunlin Shang ${ }^{\text {a }}$, Guangan Zhang ${ }^{\text {a, b,*, }}$ \\ Qunji Xue ${ }^{\text {a, b }}$ \\ ${ }^{a}$ State Key Laboratory of Solid Lubrication, Lanzhou Institute of Chemical Physics, Chinese \\ Academy of Sciences, Lanzhou 730000, China \\ ${ }^{b}$ Center of Materials Science and Optoelectronics Engineering, University of Chinese Academy of \\ Sciences, Beijing 100049, China \\ ${ }^{c}$ School of Mechanical Engineering, Yanshan University, Qinhuangdao 066004, China
}

\section{Abstract}

Demand for reduction in friction and improvement in wear resistance of moving parts propels exploration in frictional origin for amorphous carbon (a-C) film lubricating properties based on the interfacial states. Methane, as an ideal energy carrier and industrial raw material, is one of active gas. Consequently, the relations between the tribological behaviors of a-C film under methane atmosphere and load or interfacial states were discussed based on experimental and theoretical methods. Experimental results illustrated that, as the load increased, tribological system exhibited various interfacial shear strength at a load of zero and pressure dependence of the shear strength for tribological systems. And then the origin was revealed with theoretical calculation and resulted from the distributions of adsorbates across the

\footnotetext{
* Corresponding author. E-mail address: zblu@licp.cas.cn (Zhibin Lu)

* Corresponding author. E-mail address: gazhang@licp.cas.cn (Guangan Zhang)
} 
sliding interface.

Keywords: Amorphous carbon film; Tribology; Hertzian elastic contact model; first principles calculations.

\section{Introduction}

Friction exists in our daily life, resulting in nearly $20 \%$ of the total energy consumption[1]. Thus, the adverse effects of friction are reduced by covering the moving parts with solid lubrication films[2, 3]. Amorphous carbon (a-C) film, with excellent tribological performances, is widely applied in industrial field[4-6]. Nevertheless, the tribological performances of a-C film are complicated functions of test conditions and interfacial structures, leading to the empirical nature of interfacial regulation[7]. Thus, it is essential to understand the basic issue or the origin of friction based on the interfacial states.

The achievements towards interfacial states of a-C film have provided basic knowledge on regulating tribological performances of a-C film in certain conditions. Specifically, based on the factor that an atomic-scale structural mismatch between two surfaces leads to vanishing friction, an atomic-scale structural superlubricity in nitrogen atmosphere was achieved by the combination of carbonaceous materials (a-C film and fewlayer graphene) with nanometer-sized diamond particles[8]. The mechanism was the formation of nano-scrolls acting as miniature ball bearings on the sliding interface. The similar antifriction effect was found in the combination of a-C 
film with two dimensional and zero dimensional nanomaterials under certain condition[9-11]. And then, a-C film was designed with micro-nano structures, such as fullerene-like[12] and onion-like[13] structures, allowing regulation in a-C film with low-shear interface. Research on nature of surface and interface chemistry of doping a-C film during sliding in moisture condition gave the experimental information of the buried sliding interface at the nanoscale[14]. Afterwards, an appropriate silicon and oxygen were incorporated in a-C film to form a silica-like tribolayer which shielded moisture effect, thus achieved robust superlubricity of a-C film in moisture condition. Present characterization technologies can't give the information about the states of adsorbate or groups on the surfaces during sliding. It is undoubtedly a great obstacle to understanding the key concern about tribological performances of a-C film. Large-scale density functional tight-binding (DFTB) molecular dynamics (MD) simulations of diamond surfaces in various amount of confined water unveiled atomic-scale friction mechanisms of water-lubrication, showing distinct water-induced reconstruction of diamond surfaces with increasing water molecules[15]. Besides, DFT was used to analyze surface stability of diamond (111) configuration with Pandey reconstruction under various amount water and revealed the various distributions of dissociated groups on surface[16]. These theoretical calculations give the deeper insight into the interfacial structures and states for surface and interface of materials in atomic scale, allowing the further understanding about the interfacial evolution behaviors of materials during activities. Consequently, the combination of theoretical calculations and experimental technology provides an 
effective way for understanding and regulating the frictional properties of a-C film. Natural gas that consists of methane is widely used in power generation and fueling of transportation systems. In those industrial practices, it is efficient, durable, and cost-effective to improve the wear resistance properties of moving parts including fuel injectors, seal packs and pistons[17, 18]. On the other hand, methane, as a kind of active gas, gives information about the basic issue of friction for a-C film under atmosphere. Therefore, the interfacial states for a-C film in methane are explored to understand the basic issue of friction.

Here, load influences in the tribological behaviors of a-C film under methane atmosphere were explored to reveal the interfacial evolution process for film based on experimental and theoretical methods. The frictional behaviors of a-C film in methane atmosphere were attributed to adsorbates bonding with carbon dangling bonds across the sliding surface. Additionally, the distributions of adsorbates varied with the strain along perpendicular to interface, leading to the various $\alpha$ and $\tau_{0}$ for tribological systems at distinct load stages. This work provides method to optimize a-C film applied in natural gas. Meanwhile, it gives the deeper insight into the origin of friction for a-C film under atmosphere.

\section{Methodology}

\subsection{Materials}

Unbalanced magnetron sputtering ion deposition system was carried out to grow a-C film on a highly polished flat $304 \mathrm{C}$ stainless steel (SS) substrate $(3 \mathrm{~cm} \times 3 \mathrm{~cm} \times$ 
$0.3 \mathrm{~cm}$ in dimensions). And the detailed deposition procedure of film has been reported elsewhere[19]. Following the $\mathrm{Cr} / \mathrm{a}-\mathrm{C}: \mathrm{Cr}$ transition layer is a-C layer. Deposition parameters are given in Table. 1. The prepared film was approximately 2.9 $\mu \mathrm{m}$ and possessed a roughness $(\mathrm{Sa})$, which was measured by optical profilometry, of about $25 \mathrm{~nm}$. The hardness and Young's modulus, which were examined by nanoindentation measurements, were about $16.3 \pm 0.3$ and $216.7 \pm 1.5 \mathrm{GPa}$, respectively. Besides, commercially available 440 C SS ball with a hardness of $\mathrm{HRC}=55$, a Young's modulus of $\sim 200 \mathrm{GPa}$ and a surface roughness of $30 \mathrm{~nm}$, was used as a counterpart in test.

Table. 1 Deposition parameters of a-C film

\begin{tabular}{c|c|c|c}
\hline & $\begin{array}{c}\text { chamber pressure } \\
(\mathbf{P a})\end{array}$ & $\begin{array}{c}\text { target } \\
\text { current(A) }\end{array}$ & $\begin{array}{c}\text { substrate } \\
\text { pulsed bias(V) }\end{array}$ \\
\hline $\begin{array}{c}\text { Clean with Ar } \\
\text { sputtering }\end{array}$ & $3 \times 10^{-3}$ & -- & -500 \\
\cline { 1 - 2 } Transition layer & & $\mathrm{Cr}$ & -70 \\
\cline { 1 - 2 } & \multirow{2}{*}{$0.1(\mathrm{Ar})$} & 3.0 & -70 \\
\cline { 3 - 4 } & & $\mathrm{C}$ & -7.5 \\
\hline
\end{tabular}

\subsection{Friction tests}

The $440 \mathrm{C}$ SS ball with a diameter of $6 \mathrm{~mm}$ was loaded on the as-deposited a-C film at normal forces ranging from $0.2 \mathrm{~N}$ to $3 \mathrm{~N}$. And the various normal forces were increased in sequence of $0.2,0.25,0.28,0.5,1,1.75,2$ and $3 \mathrm{~N}$, corresponding to the mean Hertzian contact pressures of 254, 274, 284, 345, 434, 526, 550 and $630 \mathrm{MPa}$, respectively. These tests were run using the reciprocating model on a high vacuum tribometer (HVTRB, Anton-Paar) under a vacuum degree of $3.0 \times 10^{-3} \mathrm{~Pa}$ and a 
methane pressure of $250 \mathrm{~Pa}$. The tribometer has been reported in previous work[20]. In these tests, average speed of $2 \mathrm{~cm} / \mathrm{s}$ was obtained with a frequency of $2 \mathrm{~Hz}$ and half amplitude of $2.5 \mathrm{~mm}$ here. Then ball slid over surface of film at room temperature $(293+1 \mathrm{~K})$ for vibration cycles of 3000 . Each condition was operated three times on a fresh track with a new counterpart ball.

\section{Results}

\subsection{The friction coefficients of a-C film under various loads.}

Fig. 1 (a) displays the frictional curves of a-C film at different loads under a vacuum degree of $3.0 \times 10^{-3} \mathrm{~Pa}$. There are two identifiable types of friction behaviors: (1) 0.2 to $1 \mathrm{~N}$, (2) 1.75 to $3 \mathrm{~N}$. The curves under applied loads in range of 0.2 to $1 \mathrm{~N}$ reach its steady-state after a run-in period (1500 cycles), during which a carbonaceous transfer film is developed on the counterpart. Therefore, the steady-state friction coefficient below was recorded after 1500 cycles. However, when the normal load is above $1.75 \mathrm{~N}$, the friction coefficient fluctuates around 0.8 , and then, the film is worn out quickly. Fig. 1 (b) gives the frictional curves of the a-C film tested at various loads under a methane pressure of $250 \mathrm{~Pa}$. Each frication curve is steady after a run-in period (800 cycles), during which the friction coefficient increases to a peak quickly and then decreases drastically to a value much lower than the initial value. 

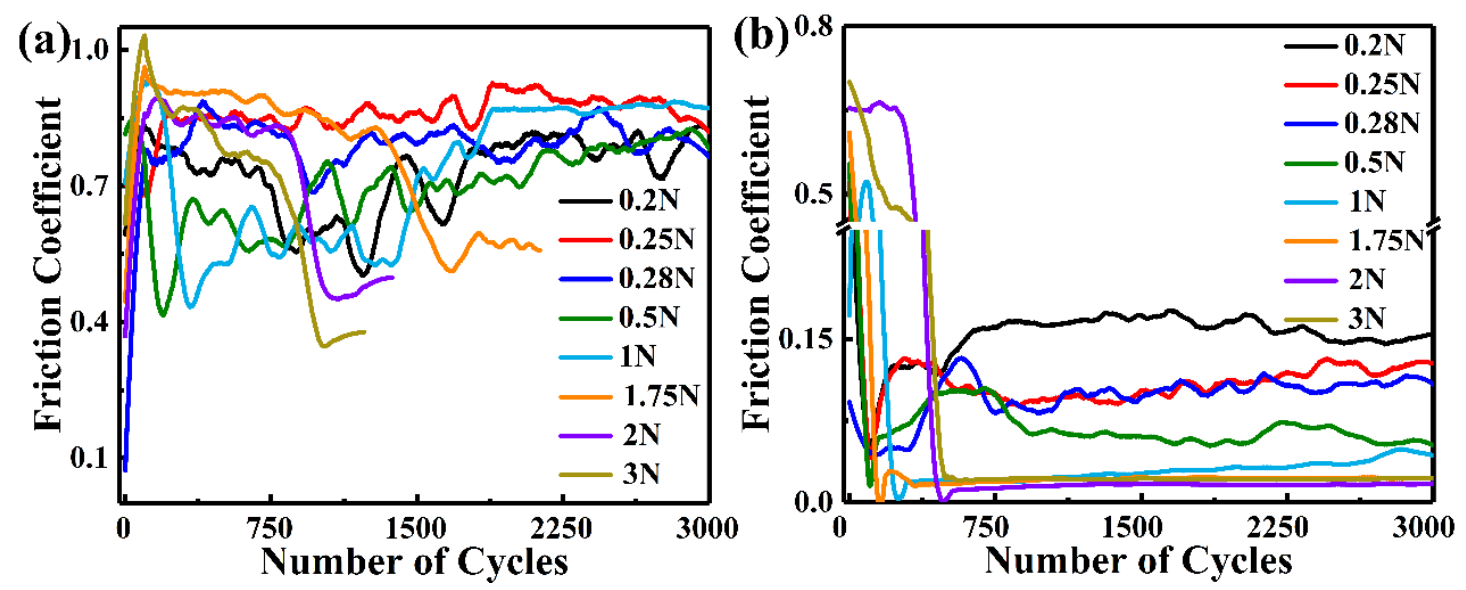

Fig.1. Friction curves of a-C film with different loads under (a) vacuum and (b) a methane pressure of $250 \mathrm{~Pa}$.

To further reveal the load influences in the frictional behaviors of a-C film, the relation between load and the average steady-state friction or friction coefficient is shown in Fig.2 (a) and Fig.2 (b), separately. As represented in Fig.2 (a), the average steady-state friction obtained in vacuum against normal load could be linearly fitted with a slope of 0.792 . However, friction increases nonlinearly with the load under a methane pressure of $250 \mathrm{~Pa}$. One can draw that there is only a slight increase in friction is produced in methane by the increased normal contact pressure from the raising of normal load, compared with the big increase at vacuum condition. Therefore, the friction coefficient is described with Hertzian elastic contact model, the relationship between friction coefficient and Hertzian pressure is given in Eq. (1).

$$
\mu=\frac{\tau_{0}}{P}+\alpha
$$

Where, $\alpha$ is the pressure dependence of the shear strength, $P$ is the Hertzian pressure, and $\tau_{0}$ is the interfacial shear strength at a load of zero. From Fig. 2(b), it is interesting to note from the result that while friction coefficient for the film under a vacuum degree of $3.0 \times 10^{-3} \mathrm{~Pa}$ is independent of inverse Hertzian pressure, the 
friction coefficient of film tested at various loads in a methane pressure of $250 \mathrm{~Pa}$ increased obviously with inverse Hertzian pressure, which is in accord with the Hertzian elastic contact model. It should be noted that increased normal load on the film can lead to a raising stress, resulting in plastic deformation, cracking and spalling of film. Here, film begins to wear off under a load of $3 \mathrm{~N}$ (Fig. S1). And it is amazing to find two stages of film's friction behavior with increasing Hertzian pressure before wearing off: (1) 254 to $284 \mathrm{MPa}$, (2) 345 to $550 \mathrm{MPa}$. At the first stage, a slope of $149 \mathrm{MPa}$ is given by linear fitting of the average steady-state friction coefficient against inverse Hertzian pressure. Then a slope $\sim 37 \mathrm{MPa}$ is obtained with the decrease of inverse Hertzian pressure. It indicates that the main factor governing the tribological performances of films changes with loads, leading to the different interface evolution with different Hertzian pressure.
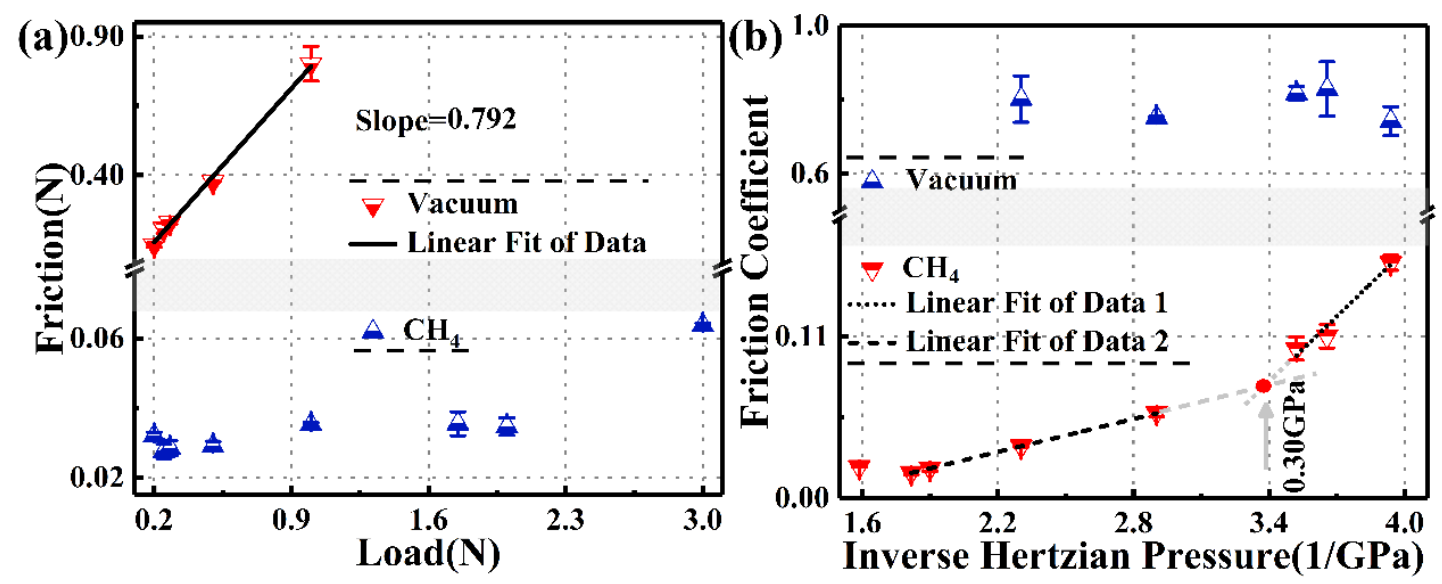

Fig.2. Summaries for (a) the average steady-state friction and (b) the average steady-state friction coefficient for a-C film with different loads in vacuum and a methane pressure of $250 \mathrm{~Pa}$.

\subsection{Structural characterization of sliding surfaces}

Structural analyses of sliding interfaces provide information about mechanism 
for load influences in the frictional behaviors of a-C film in methane atmosphere. Therefore, the structures for sliding surfaces were examined via Raman spectrum and transmission electron microscopy (TEM). Figs. 3 (a) - (d) display the select regions of Raman spectrum characterization and Figs. 3 (e) - (f) give the Raman spectra of wear tracks and wear scars. One can draw that pristine and worn films present typical characteristics of a-C film that a broad peak in the frequency ranging from $900-1800$ $\mathrm{cm}^{-1}$ is observed. Then the peak could be fitted into two peaks (D peak and G peak) by Gaussian method. As we all know, D peak results from the breathing mode of $\mathrm{sp}^{2}$ $\mathrm{C}$ atoms in rings while the $\mathrm{G}$ peak results from the stretching vibration of all pairs of $\mathrm{sp}^{2} \mathrm{C}$ atoms in both rings and chains. Fig. S2 displays that ID/IG of a-C film increases after sliding, illustrating the graphitization of a-C film. From Fig. 3 (f), it is obvious that transfer films exhibit the typical characteristics of graphitized carbonaceous material. 

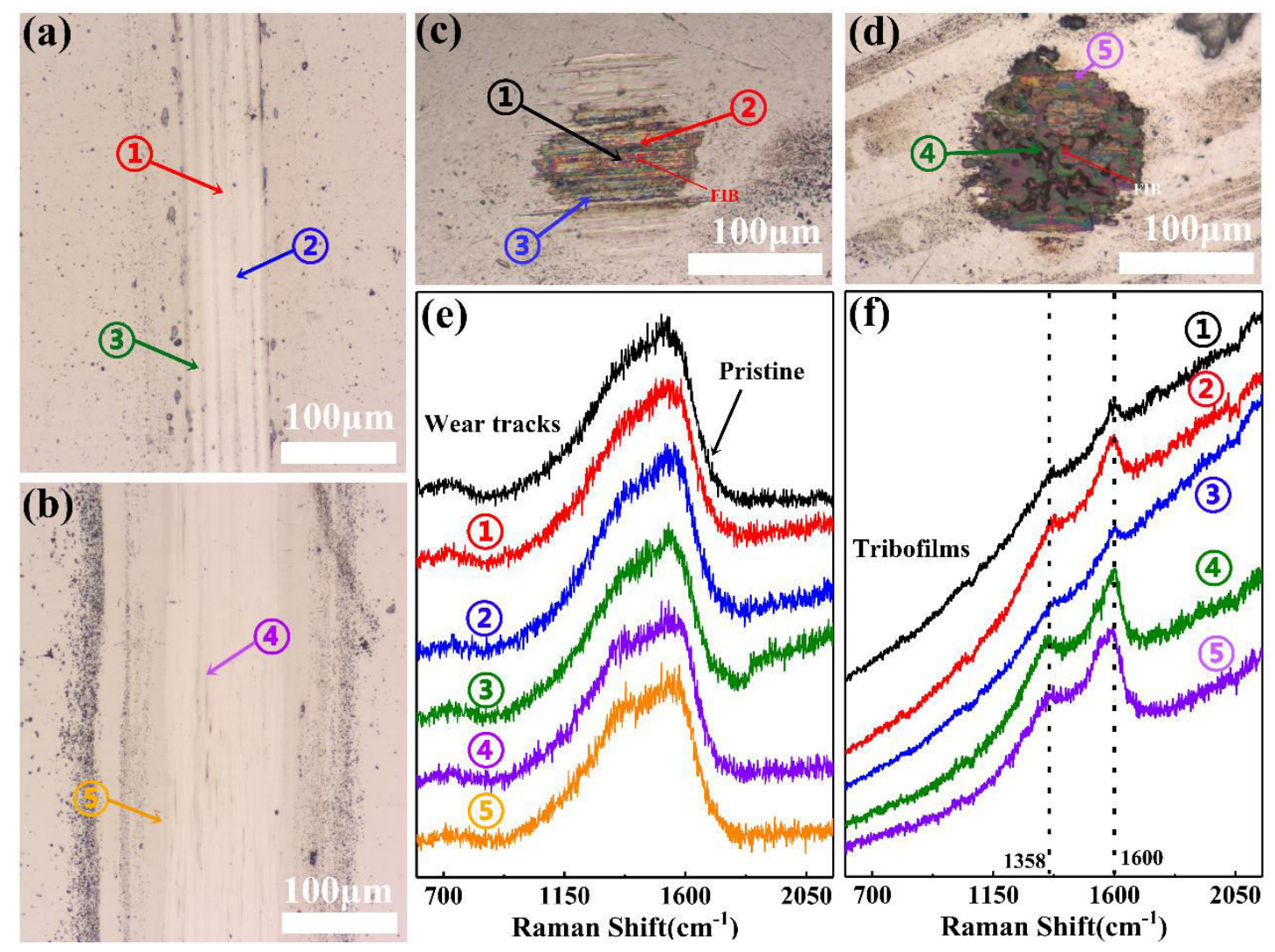

Fig.3. Optical photographs and Raman spectra for worn surfaces of films and balls under different loads: (a), (c), (e) $0.25 \mathrm{~N}$ and (b), (d), (f) $1 \mathrm{~N}$.

The TEM samples of transfer film were prepared with focused ion beam (FIB) technology to further analyze the structure of sliding interface. And the results are given in Fig. 4. The microstructure of transfer film in Fig. 4 (a) illustrates the tribofilm under a load of $0.25 \mathrm{~N}$ consists of several layers containing a"tumor-like" amorphous layer near the sliding interface. And it is obvious that there are many nanoclusters induced by accumulation of "tumor-like" carbonaceous material in tribofilms. From Fig. 4 (b), the tribofilm under a load of $1 \mathrm{~N}$ is found to contain a rodlike amorphous layer near the sliding interface. No nanocrystals are observed in the transfer film under the two loads. Additionally, two tribofilms possess the similar amorphous outmost working layer. These results illustrate the differences in frictional 
behaviors of a-C film with various loads lay in gas adsorption.
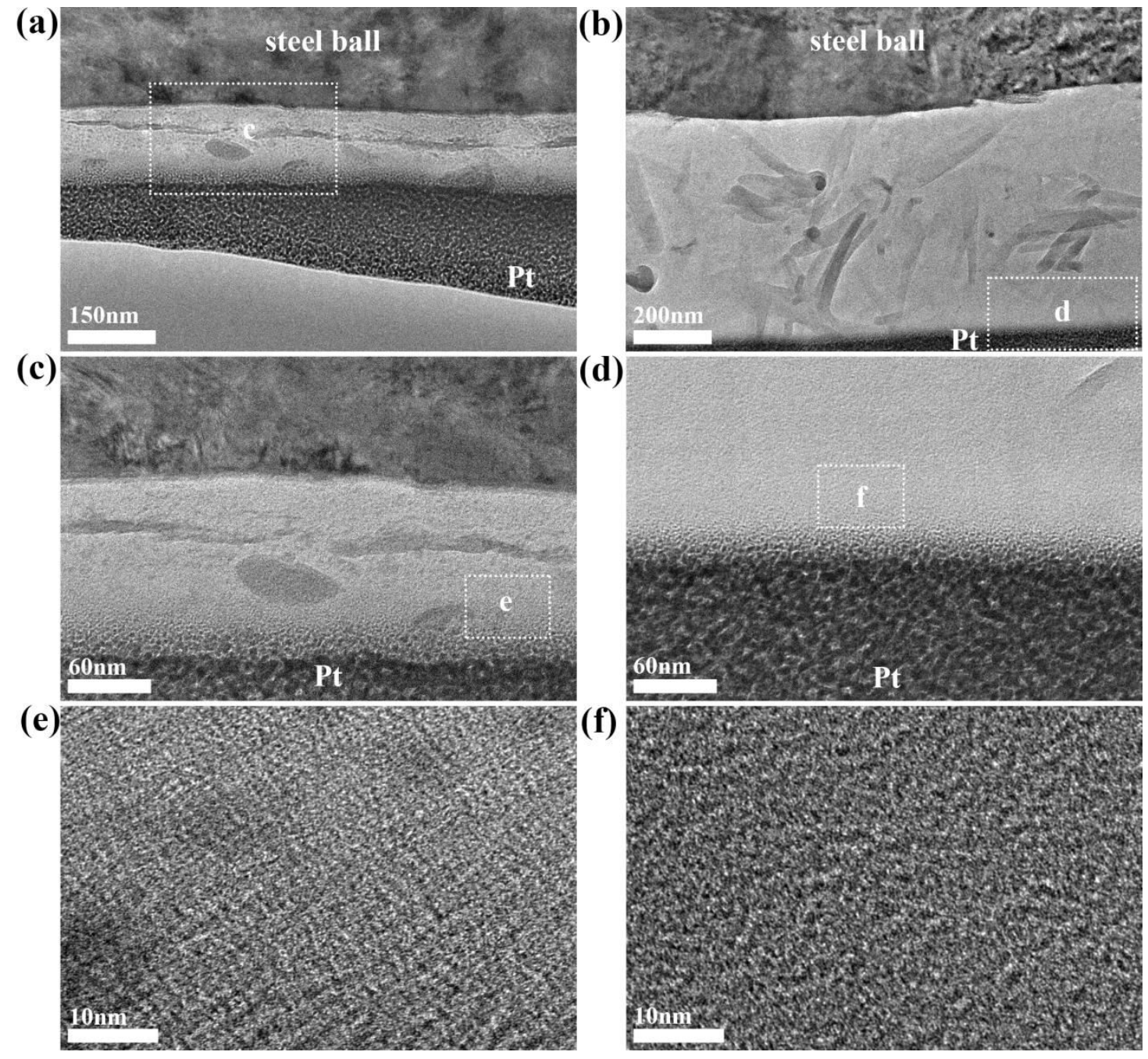

Fig. 4. The TEM observation of transfer films on counterpart balls after sliding tests under a load of (a), (c), (e) $0.25 \mathrm{~N}$ and (b), (d), (f) $1 \mathrm{~N}$. Select regions of local enlarged images are marked.

\subsection{Interfacial Analysis based on first principles calculations}

First-principles calculations were carried out to obtain friction between sliding surfaces in order to gain the deeper understanding about the frictional mechanism for a-C film in methane atmosphere. Based on above experimental results, the model, as shown in Fig. 5 and Figs. 7 - 8, was constructed to give a deeper insight into the processes of interfacial evolution process at atomic scale[21, 22]. Figs. 5 (b) - (d) 
present the computational process about the effects of stress induced by compressive strain on structures. According to the formula about compressive strain: $\varepsilon=\frac{c_{1}-c_{0}}{c_{0}}$, one can obtain the structures and stress under various compressive strain by optimizing the configurations possessing reduced and fixed length of $c_{0}$. The calculated progress was based on density functional theory (DFT) approach, which was implemented by the CASTEP package within the Materials Studio framework[23]. During calculation process, the exchange-correlation energy was characterized via the generalized gradient approximation (GGA) in the form of the Perdewe-Burkee-Ernzerhof (PBE) function[24]. Additionally, A plane-wave cutoff energy of $650 \mathrm{eV}$ and Monkhorst-Pack k-point mesh density of $(5 \times 5 \times 1)$ were adopted in this work. From Fig. 5 (c), methane molecule dissociates into methyl and hydrogen which bond with carbon dangling bonds across the interface of diamond (111).

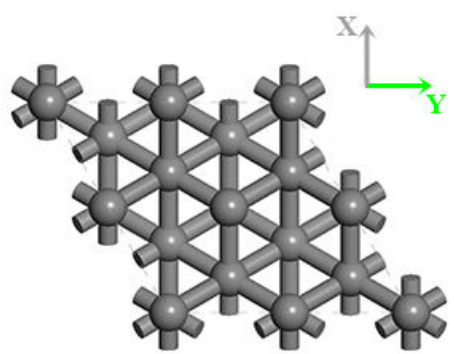

Top layer

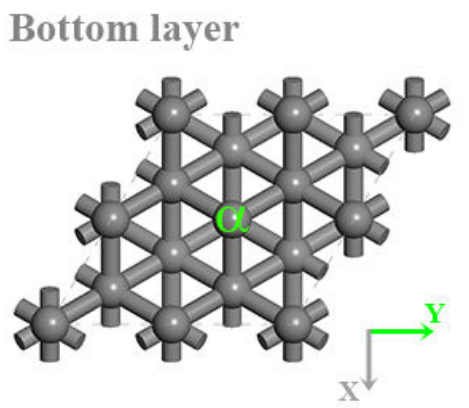

(a)

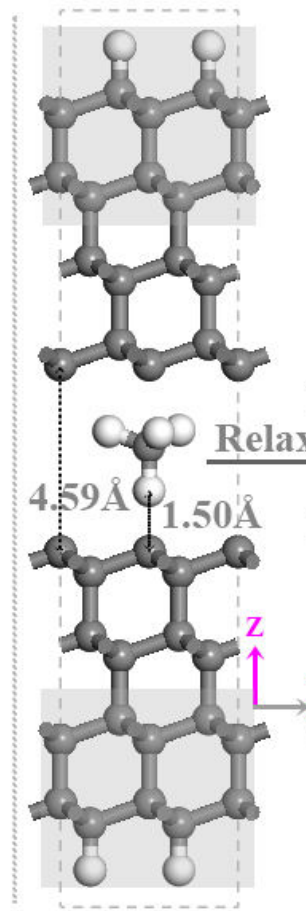

(b)

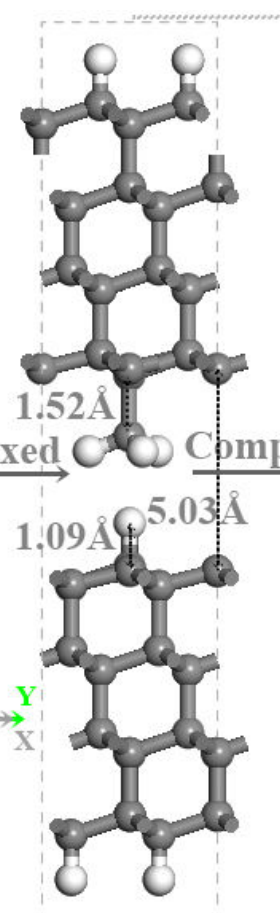

(c)

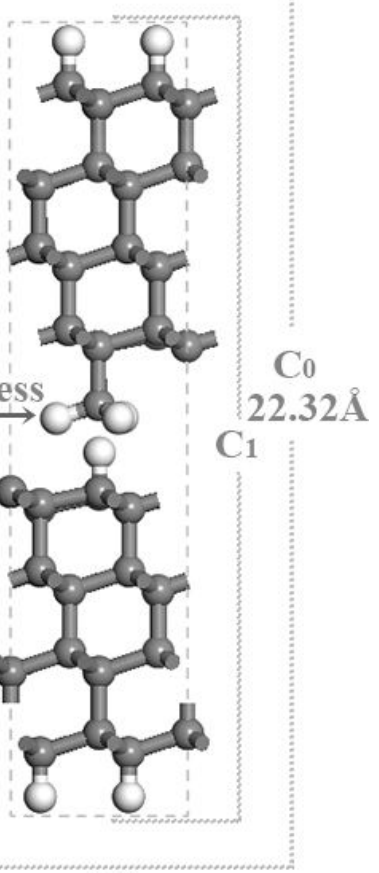

(d) 
Fig. 5. Configurations used for calculation of stress: (a) and (b)The initial configuration at $\varepsilon=0$, (c) the relaxed configuration at $\varepsilon=0$, (d) the application direction of strain. And a methane molecule is placed above the position marked $\alpha$. The part in shadow is fixed to ensure bulk of the slabs. In addition, atom type is represented by sphere color: gray $=$ carbon, white $=$ hydrogen .

Fig. 6 (a) gives stress-strain relation of diamond (111)/diamond (111) configuration under methane. The stress decreases with the increasing strain. As shown in Fig. 6 (b), the strain energy rises and its first derivative reduces with the increasing strain. It should be noted that strain energy is obtained by subtracting the energy of configuration under strain from the energy of configuration under equilibrium state. It is well-known that there are two types of deformation for materials during compression process. The form of deformation belongs to elastic deformation at low applied, with increasing applied strain, plastic deformation occurs. The plastic deformation sets in after the second yielding point where the strain energy reaches its maximum value. Of causes, there are the first yielding point, where the first derivative of strain energy reaches its minimum value. In this case, high strain is observed with small compression. No yielding point of configurations is observed at $-0.135 \leq \varepsilon \leq 0$, illustrating the elastic deformation of systems. 

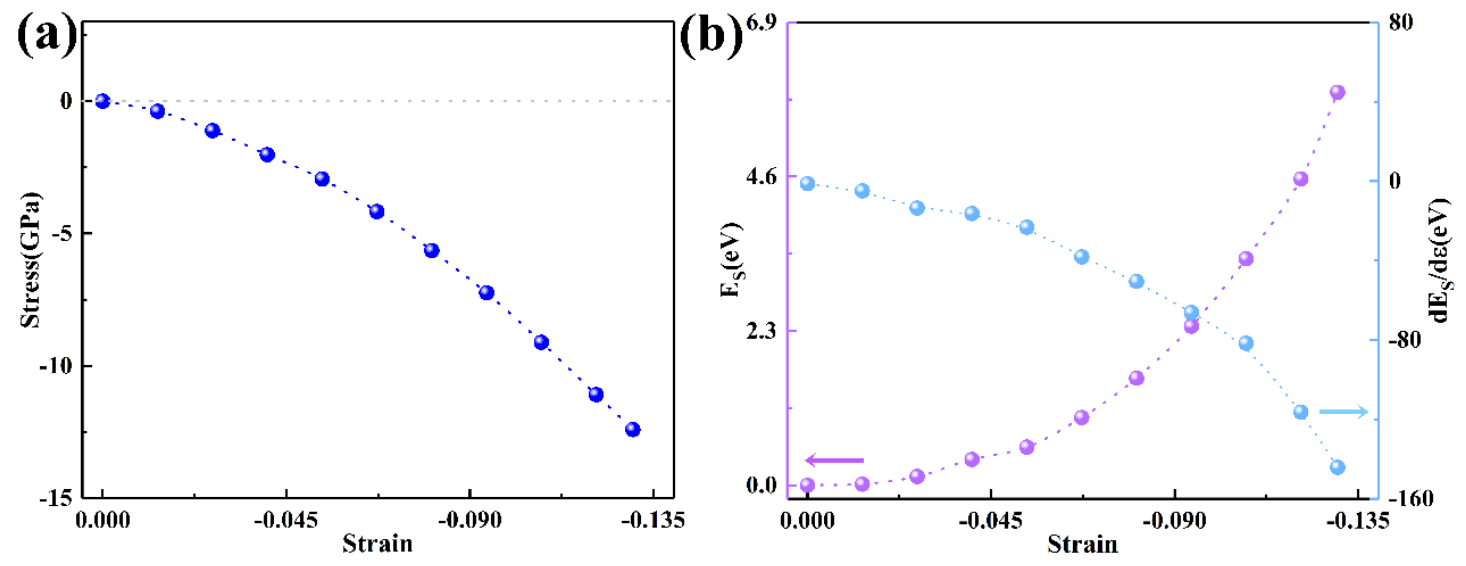

Fig. 6. (a) Variation of stress for configurations. (b) Strain energy versus strain for configurations and its first derivation to strain.

Consequently, the relaxed configurations under $\varepsilon=0,0.04,0.08,0.12$ were enlarged to eliminate the influence of the boundary and further explore the interfacial evolution processes for a-C film in methane via molecular dynamics (MD) simulations within first-principles calculation. The constant volume and energy (NVE) were carried out to conduct MD simulations with an initial temperature of $300 \mathrm{~K}$. A plane-wave cutoff energy of $380 \mathrm{eV}$ and Grimme method were adopted to $\mathrm{MD}$ simulations. In addition, the MD time step was 1.0 fs and the total MD simulation time was 1.0 ps. We want to state that, compared with other configurations (Fig. S3), the configuration given in Fig. 7 possesses the lowest energy. Therefore, the configuration given in Fig. 8 was chosen. Fig. 7 displays the initial and reconstructed configurations for MD simulations. One can draw that dissociation groups bonded to carbon dangling bonds across the top and bottom layers are opposite at low applied, with increasing applied strain, they are staggered. After that, the methyl groups dissociate and carbon dangling bonds emerge under further compression. After the MD simulations of configurations, the top layer slides horizontally relative to the 
bottom one, allowing the analysis for sliding pathway of tribological systems under various strain. Fig. 8 presents the schematic diagram of sliding pathway. Every sliding pathway is divided into forty-one steps. Interactions between layers are measured with separation work $W_{\text {sep }}$ and obtained with Eq. (3).

$$
W_{\text {sep }}=\frac{\left(E_{12}^{t o t}-E_{S}^{t o t}\right)}{A}
$$

Where $E_{12}^{\text {tot }}$ are the total energy of the configuration in while the distance between the top and bottom layers is $10 \AA$, and $E_{S}^{\text {tot }}$ are the total energy of the configuration after sliding and $A$ are the interfacial area. Then, deviations between the highest energy $\left(E_{\max }\right)$ and lowest energy $\left(E_{\text {min }}\right)$ of the configurations in the sliding pathways are calculated to measure the sliding pathways under a certain load. Here, the load is obtained by fixing the length of $c_{0}$ to remain interlayer distance. Relative sliding between layers must overcome the potential energy corrugation on the sliding pathway, and thus the friction behavior of system is closely related to the maximum potential energy barrier. Consequently, the average friction of system is calculated with Eq. (4).

$$
F=\frac{E_{\max }-E_{\min }}{\Delta s}
$$

Where $\Delta s$ is the sliding distance. It should be stated that only one sliding pathway under $\varepsilon=0.12$ is analyzed due to the emergence of carbon dangling bonds. 

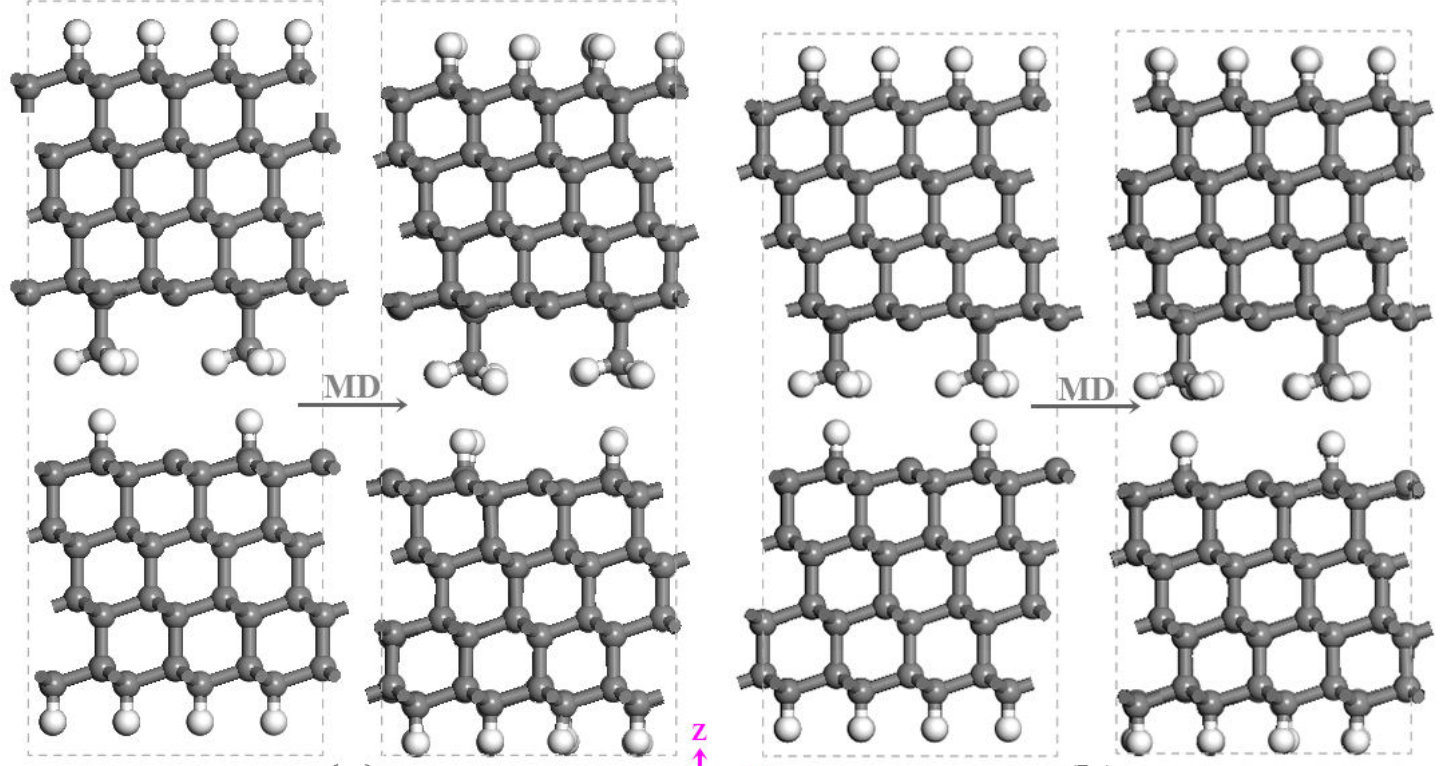

(a)

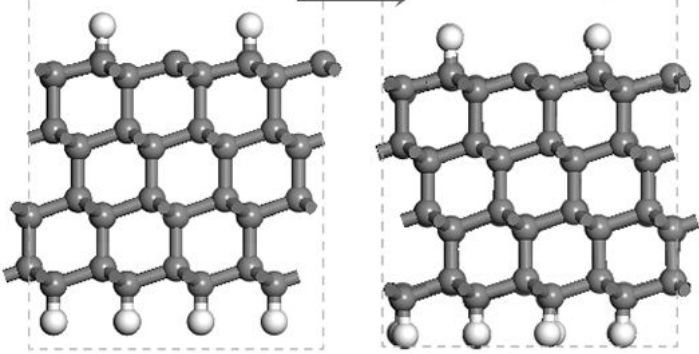

(b)
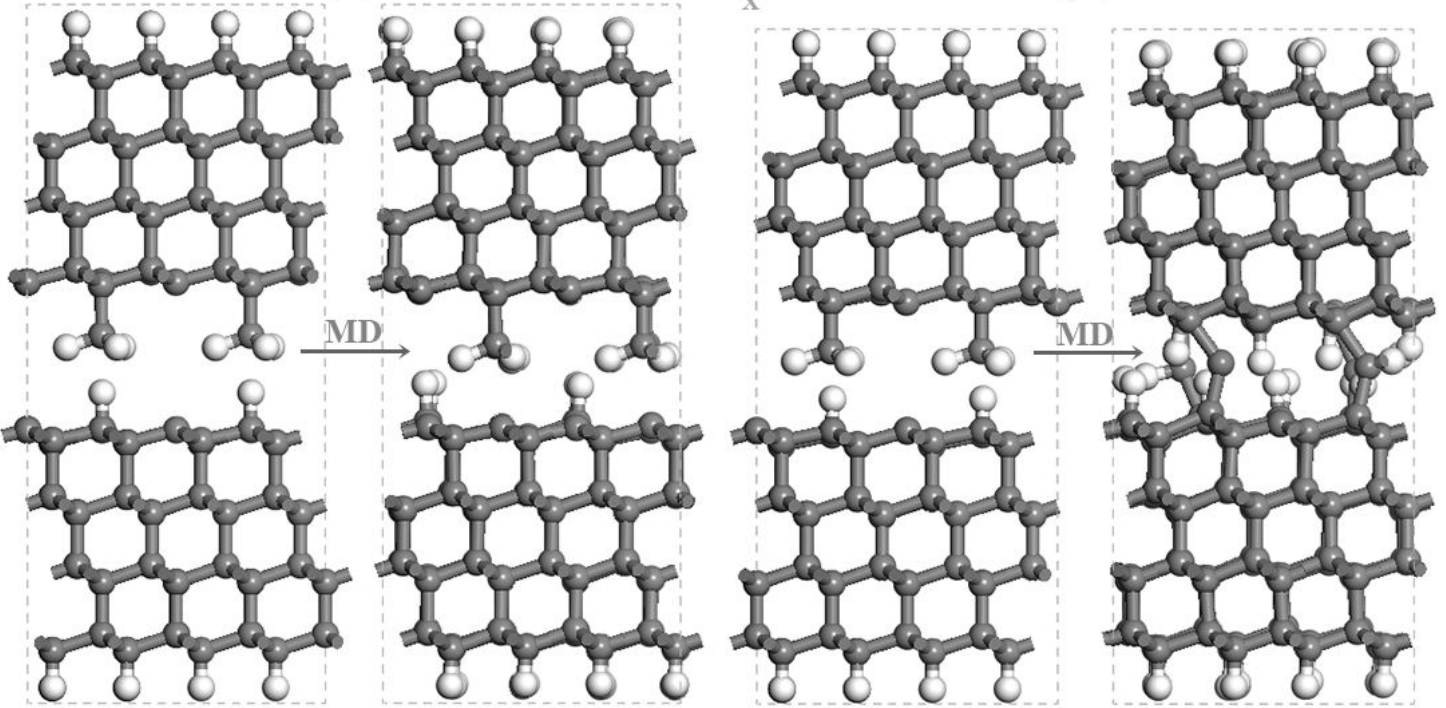

(c)
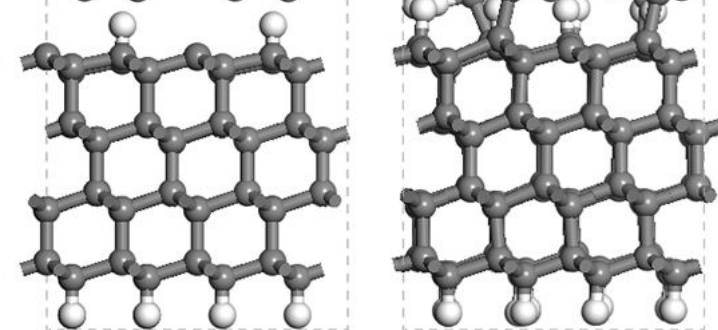

(d)

Fig. 7. The initial and corresponding reconstructed microstructures of configurations under various strain: (a) $\varepsilon=0$, (b) $\varepsilon=0.04$, (c) $\varepsilon=0.08$, (d) $\varepsilon=0.12$. 


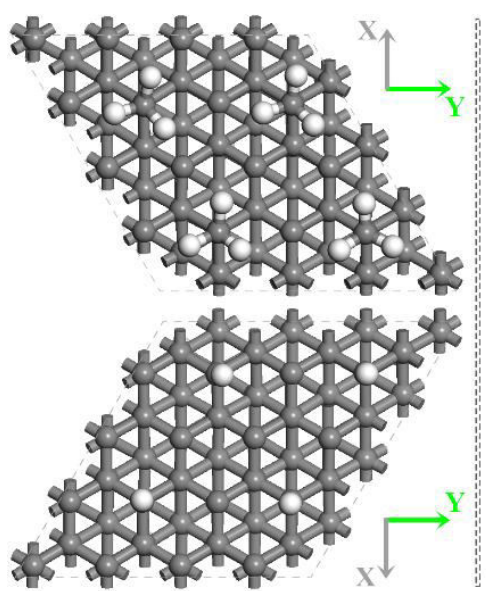

(a)

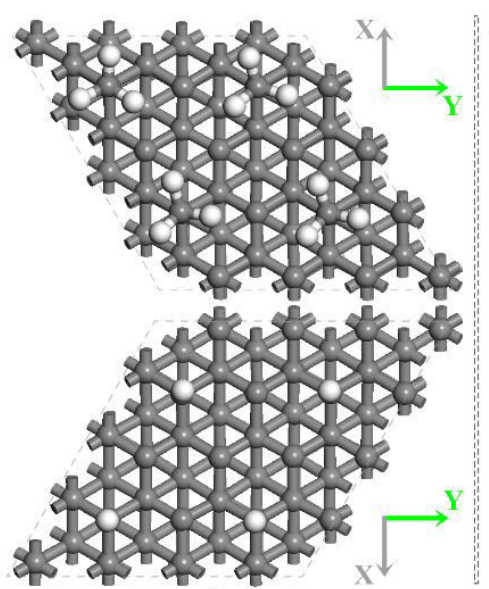

(b)

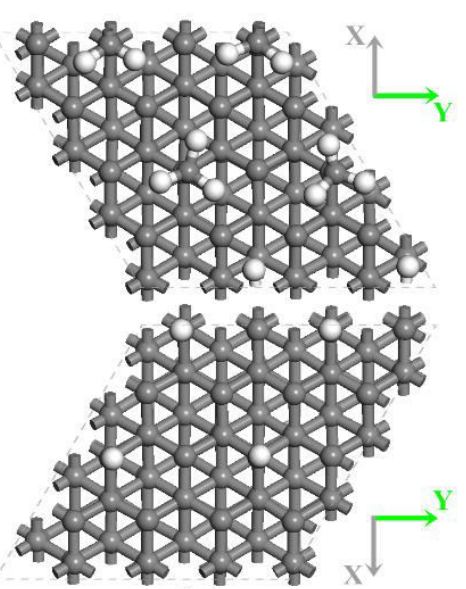

(c)

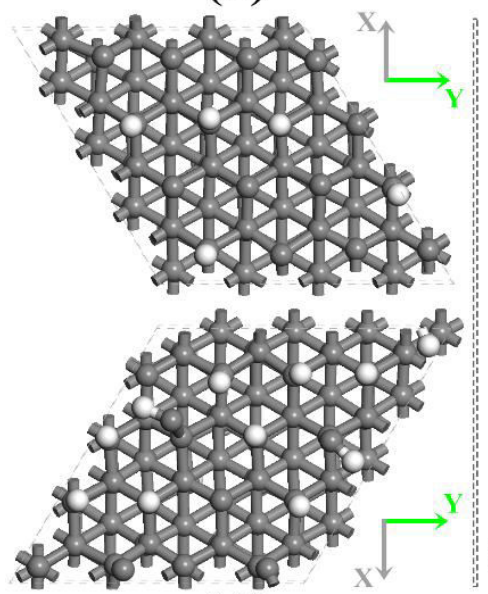

(d)

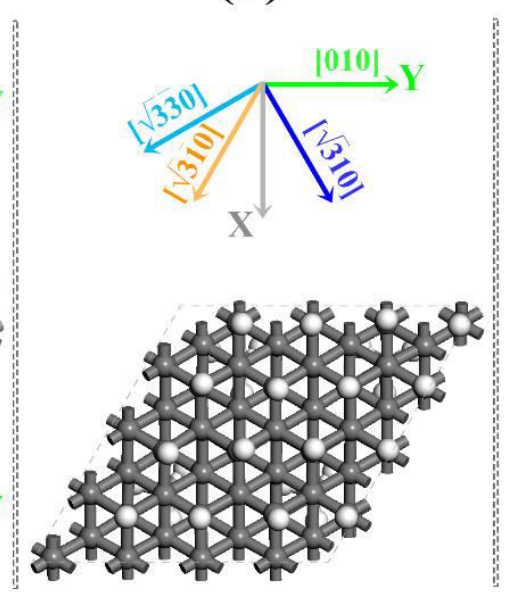

(e)

Fig. 8. The schematic diagram to visually present the configurations and sliding pathways of tribological systems: the configurations under (a), (e) $\varepsilon=0$, (b) $\varepsilon=$ 0.04 , (c) $\varepsilon=0.08$, (d) $\varepsilon=0.12$, as well as the sliding pathways of configurations viewed along the $\left[\begin{array}{lll}0 & 0 & 1\end{array}\right]$ direction.

The $W_{\text {sep }}$ and $F$ are presented in Fig. 9. It is obvious that the potential energy corrugation on the sliding pathway increases with strain. Meanwhile, relative sliding of each system along the $\left[\begin{array}{lll}\sqrt{3} & \overline{1} & 0\end{array}\right]$ direction possesses the minimum friction among those sliding pathways. A great increase in friction of tribological system is observed under $\varepsilon=0.12$, resulting from the adhesion between sliding interface. Combined with Fig. 8, it can be seen that the adsorbates across the sliding surfaces arrange from 
head-on mode to side-by-side mode with the increased strain during sliding. As interfacial adsorbates slide head to head at a low load, the adsorbates on top and upper and lower layers encounter each other, leading to the vibrational excitation of adsorbates. Then this energy is imparted from adsorbates to the rest of the lattice subsequently. This energy dissipation results in the friction of tribological systems [25]. When adsorbates on the top layer travel between adjacent adsorbate rows of bottom layer, in addition to the interaction with the bottom layer adsorbates, the adsorbates on the top layer interact with the carbon dangling bonds of second layer. Consequently, adsorbates with different arrangements on sliding interfaces, which are induced by various strain, interact in different modes, and thus the tribological system is in accord with the Hertzian elastic contact model corresponding to distinct stages with various $\alpha$ and $\tau_{0}$. 

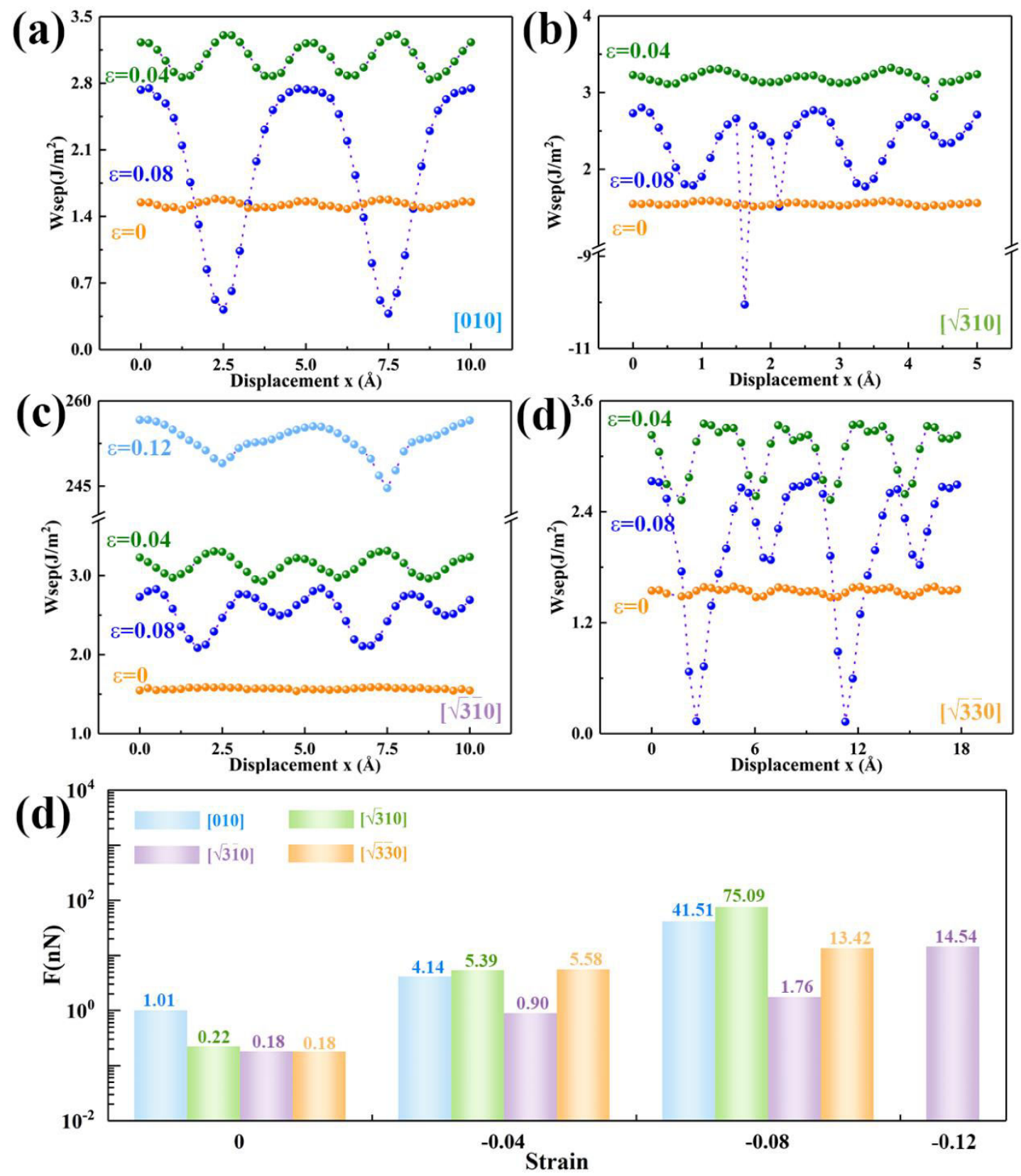

Fig. 9. Separation work and average friction of configurations sliding along different pathways: (a)-(d) separation work and (e) average friction.

\section{Discussion}

Previous study about the shear properties of Langmuir-Blodgett layers during sliding stated the relation between the shear strength of L-B layer and the contact pressure and revealed the origin with mathematical model of activated processes developed by Eyring[26]. The mathematical model assumes that the motion of 
molecules has to overcome potential barriers $\left(Q^{\prime}\right)$ from their neighbours by shear stress and random thermal. And the product of the Boltzmann factor and the effective vibration frequency of molecules $v$ is the average time $\left(t^{\prime}\right)$ for molecules passing through energy barrier, as shown in Eq. (5).

$$
\frac{1}{t^{\prime}}=v \exp \left\{\frac{-\left(Q^{\prime}+\Omega P-\phi \tau\right)}{k T}\right\}
$$

Where $Q^{\prime}+\Omega P-\phi \tau$ is the energy barrier, $\Omega$ and $\phi$ represent the pressure and stress activation volumes separately. The average velocity $\bar{V}$ of molecules under the potential gradient is obtained by Eq. (6).

$$
\bar{V}=2 v \exp \left\{\frac{-\left(Q^{\prime}+\Omega P\right)}{k T}\right\} \sinh \left(\frac{\phi \tau}{k T}\right)
$$

Where $b$ represents the distant by which the regular series of barriers are separated. And then, the sliding velocity $V$, which is proportional to $\bar{V}$, can be obtained with Eq. (7) by introducing an unknown velocity $V_{0}$.

$$
V=2 V_{0} \exp \left\{\frac{-\left(Q^{\prime}+\Omega P\right)}{k T}\right\} \sinh \left(\frac{\phi \tau}{k T}\right)
$$

The Ea. (7) can be written as Eq. (8) due to $\frac{\phi \tau}{k T}<1$.

$$
V=2 V_{0} \exp \left\{\frac{-\left(Q^{\prime}+\Omega P-\phi \tau\right)}{k T}\right\}
$$

It is equivalent to Eq. (9)

$$
\tau=\frac{k T}{\phi} \ln \left(\frac{V}{V_{0}}\right)+\frac{1}{\phi}\left(Q^{\prime}+\Omega P\right)
$$

Therefore, the relationship between friction coefficient and Hertz contact pressure, as given in Eq. (10).

$$
\mu=\frac{1}{P} \cdot \frac{1}{\phi}\left\{k T \ln \left(\frac{V}{V_{0}}\right)+Q^{\prime}\right\}+\frac{\Omega}{\phi}
$$

During sliding, the gas adsorption across interface is dynamic stable, resulting in the shear-induced self-assembled layer on the sliding interface. Therefore, Eq. (10) is 
suitable to sliding interface of a-C film under atmosphere. The potential barrier $\left(Q^{\prime}\right)$ or activation energy of the process is almost the same for the various loads. Therefore, for the experimental results above, the stress and pressure activation volumes ( $\phi$ and $\Omega$ ) in stage (1) is lower than those in stage (2). Combined with the calculated results, the increasing energy barrier during sliding is found.

In summary, the interfacial evolution process of a-C film with various loads in methane atmosphere is revealed. During sliding, methane molecule dissociates into methyl and hydrogen, bonding to the carbon dangling bond across the sliding interface. The applied load affects the distribution models of adsorbates, leading to the different origin of energy dissipation for tribological systems. Specifically, the distribution of adsorbates on top and bottom surfaces transfers from head-on mode to side-by-side mode with the increasing load, as shown in Fig. 10. Consequently, the origin of energy dissipation changes from the interaction between adsorbates to interaction between adsorbates and carbon dangling bonds or adsorbates, resulting in the distinct $\alpha$ and $\tau_{0}$ for tribological systems under various stages of film's tribological performance. 


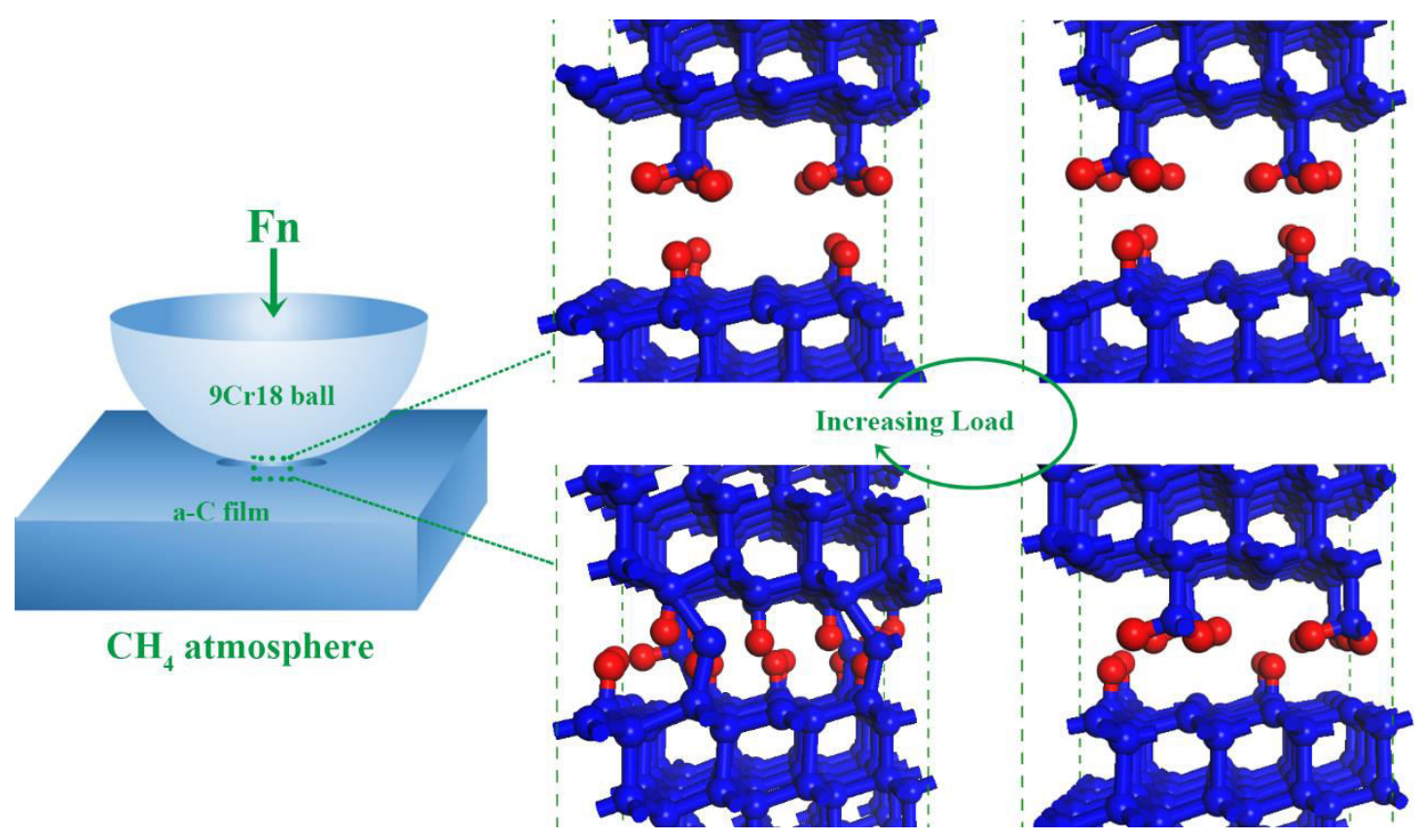

Fig. 10. Schematic description of lubricant mechanism occurring in sliding. Atom types is represented by color of the sphere: blue=carbon, red=hydrogen.

\section{Conclusions}

The frictional behaviors of a-C film in methane atmosphere are explored with the load effects based on experimental and theoretical methods. It is found that the contact of a-C film and ball under various loads is in accord with the Hertzian elastic contact model. Additionally, as load increasing, there are various $\alpha$ and $\tau_{0}$ for tribological systems. Theoretical analyses of interfacial evolution processes give the deeper insight into the interfacial interaction at the atomic scale and reveal factors that determine the frictional behaviors of a-C film in methane atmosphere. It is the different arrangements of adsorbates on top and bottom sliding surfaces under various loads lead to different kinds of groups, which interact with each other, and thus affect the load dependence of a-C film in methane atmosphere. 


\section{Acknowledgments}

This work was supported by National Natural Science Foundation of China [grant number 11972344], CAS "Light of West China" Program.

\section{Declaration of Competing Interest}

The authors declare that they have no known competing financial interests or personal relationships that could have appeared to influence the work reported in this paper.

\section{References}

[1] Zhang, S., Ma, T., Erdemir, A., Li, Q. Tribology of two-dimensional materials: From mechanisms to modulating strategies. Materials Today 26:67-86 (2019).

[2] Chen, X., Li, J. Superlubricity of carbon nanostructures. Carbon 158:1-23 (2020).

[3] Pilotti, B., Prieto, G., Juan, A., Faccio, R., Broitman, E., Dennehy, M., et al. Bi2S3 and MoS2 Soft Coatings: A Comparative Study of Their Frictional Behavior Under Different Humidity Levels, Normal Loads, and Sliding Speeds. Tribology Letters 69 (2021).

[4] Chen, L., Wei, X., Zhang, G., Shang, L., Lu, Z., Nie, X., et al. Probing the tribological performances of hydrogenated amorphous carbon film in methane atmosphere based on Hertzian elastic contact model. Tribology International 155:106790 (2021). 
[5] Rani, R., Panda, K., Kumar, N., Sankaran, K.J., Ganesan, K., Lin, I.N. Tribological Properties of Ultrananocrystalline Diamond Films in Inert and Reactive Tribo-Atmospheres: XPS Depth-Resolved Chemical Analysis. The Journal of Physical Chemistry C 122:8602-8613 (2018).

[6] Wu, J., Wu, G., Kou, X., Lu, Z., Zhang, G., Wu, Z. Probing Tribological Behaviors of Cr-DLC in Corrosion Solution by Tailoring Sliding Interface. Tribology Letters 68 (2020).

[7] Baykara, M.Z., Vazirisereshk, M.R., Martini, A. Emerging superlubricity: A review of the state of the art and perspectives on future research. Applied Physics Reviews 5:041102 (2018).

[8] Berman, D., Deshmukh, S.A., Sankaranarayanan, S.K.R.S., Erdemir, A., Sumant, A.V. Macroscale superlubricity enabled by graphene nanoscroll formation. Science 348:1118-1122 (2015).

[9] Berman, D., Narayanan, B., Cherukara, M.J., Sankaranarayanan, S., Erdemir, A., Zinovev, A., et al. Operando tribochemical formation of onion-like-carbon leads to macroscale superlubricity. Nat Commun 9:1164 (2018).

[10] Huang, P., Qi, W., Yin, X., Choi, J., Chen, X., Tian, J., et al. Ultra-low friction of a-C:H films enabled by lubrication of nanodiamond and graphene in ambient air. Carbon 154:203-210 (2019).

[11] Mutyala, K.C., Wu, Y.A., Erdemir, A., Sumant, A.V. Graphene - MoS2 ensembles to reduce friction and wear in DLC-Steel contacts. Carbon 146:524-527 (2019).

[12] Wang, Y., Gao, K., Zhang, B., Wang, Q., Zhang, J. Structure effects of sp2-rich 
carbon films under super-low friction contact. Carbon 137:49-56 (2018).

[13] Xu, J., Chen, X., Grützmacher, P., Rosenkranz, A., Li, J., Jin, J., et al. Tribochemical Behaviors of Onion-like Carbon Films as High-Performance Solid Lubricants with Variable Interfacial Nanostructures. ACS Applied Materials \& Interfaces 11:25535-25546 (2019).

[14] Chen, X.C., Yin, X., Qi, W., Zhang, C.H., Choi, J., Wu, S.D., et al. Atomic-scale insights into the interfacial instability of superlubricity in hydrogenated amorphous carbon films. Sci Adv 6 (2020).

[15] Kuwahara, T., Moras, G., Moseler, M. Friction Regimes of Water-Lubricated Diamond (111): Role of Interfacial Ether Groups and Tribo-Induced Aromatic Surface Reconstructions. Phys Rev Lett 119:096101 (2017).

[16] Levita, G., Kajita, S., Righi, M.C. Water adsorption on diamond (111) surfaces: an ab initio study. Carbon 127:533-540 (2018).

[17] Ramirez, G., Eryilmaz, O.L., Fatti, G., Righi, M.C., Wen, J., Erdemir, A. Tribochemical Conversion of Methane to Graphene and Other Carbon Nanostructures: Implications for Friction and Wear. ACS Applied Nano Materials 3:8060-8067 (2020). [18] Chen, L., Wu, J., Lu, Z., Shang, L., Zhang, G., Xue, Q. Probing tribological performances of hydrogenated amorphous carbon film applied in methane by structural modification with boron. Wear 470-471:203610 (2021).

[19] Chen, L., Cao, X., Lu, Z., Wang, Y., Zhang, G., Xue, Q. Improving the tribological properties of diamond-like carbon film applied under methane by tailoring sliding interface. International Journal of Refractory Metals and Hard 
Materials 94:105380 (2021).

[20] Chen, L., Wang, J., Shang, L., Lu, Z., Wu, Z., Zhang, G. Gas phase lubrication on diamond-like carbon film: Tribochemical reactions under isobutane condition. Tribology International 133:152-159 (2019).

[21] Marks, N.A. Evidence for subpicosecond thermal spikes in the formation of tetrahedral amorphous carbon. Physical Review B 56:2441-2446 (1997).

[22] Luo, X.G., Liu, Z.Y., Xu, B., Yu, D.L., Tian, Y.J., Wang, H.T., et al. Compressive Strength of Diamond From First-Principles Calculation. J Phys Chem C 114:17851-17853 (2010).

[23] Clark, S.J., Segall, M.D., Pickard, C.J., Hasnip, P.J., Probert, M.J., Refson, K., et al. First principles methods using CASTEP. Z Kristallogr 220:567-570 (2005).

[24] Perdew, J.P., Burke, K., Ernzerhof, M. Generalized gradient approximation made simple. PHYSICAL REVIEW LETTERS 77:4 (1996).

[25] Harrison, J.A., White, C.T., Colton, R.J., Brenner, D.W. Effects of Chemically-Bound, Flexible Hydrocarbon Species on the Frictional-Properties of Diamond Surfaces. J Phys Chem-Us 97:6573-6576 (1993).

[26] Briscoe, B.J., Evans, D.C.B. The shear properties of Langmuir-Blodgett layers. Proc R Soc Lond A 380:18 (1982). 


\section{Supplementary Files}

This is a list of supplementary files associated with this preprint. Click to download.

- SupplementaryMaterial.docx 